\title{
Optische Anregung von Gassensoren: Raumtemperatur Ozonsensoren auf Basis von mesoporösem Indiumoxid
}

\author{
Thorsten Wagner \\ Department Chemie, Universität Paderborn, Paderborn \\ Tel.: 05251-602486, e-mail: thorsten.wagner@upb.de
}

\section{Kurzfassung}

Im folgenden Artikel werden Ergebnisse zur Raumtemperatursensorik mit optisch aktiviertem nanostrukturiertem Indiumoxid $\left(\operatorname{In}_{2} \mathrm{O}_{3}\right)$ präsentiert.

Optische Aktivierung von Metalloxiden zur Steigerung bzw. Aktivierung der Reaktivität auf Gase ist auf dem Gebiet der heterogenen Katalyse Gegenstand der aktuellen Forschung. Die beobachteten Effekte wie z.B. erhöhte Katalysatoraktivität sind auch auf dem Gebiet der halbleiterbasierten resistiven Gassensoren von hohem Interesse. Allerdings existieren im Bereich der Sensorik bisher wenig systematische Studien zu diesem Thema.

Wir präsentieren hier am Beispiel von nanostrukturiertem $\operatorname{In}_{2} \mathrm{O}_{3}$ die Übertragung von Konzepten aus der Katalyse in die Gassensorik. Durch optische Aktivierung in Kombination mit einer definierten Nanostruktur wird halbleiterbasierte Ozonsensorik bei Raumtemperatur ermöglicht.

\section{Einleitung}

Indiumoxid bietet vorteilhafte Eigenschaften als sensitives Material für resistive Halbleitergassensoren. Im speziellen ist es gut für die Detektion von oxidierenden Gasen in geringen Konzentrationen geeignet [1,2]. Bei niedrigen Betriebstemperaturen $\left(<150{ }^{\circ} \mathrm{C}\right)$ zeigen die Wirkschichten nahezu keine Reaktion auf reduzierende Gase, sind somit selektiv auf Gase wie z.B. $\mathrm{O}_{3}$ oder $\mathrm{NO}_{2}$ [3]. $\mathrm{In}_{2} \mathrm{O}_{3}$ 
ist deshalb prinzipiell ein geeignetes Material für zuverlässige Ozonsensoren im ppb-Bereich im Umweltmonitoring bei niedrigen Betriebstemperaturen. Allerdings sind die Reaktionszeiten von herkömmlichen, granularen Wirkschichten bei Raumtemperatur hoch, die Regeneration erfolgt langsam und die Signale sind nicht stabil. Es ist bekannt, dass optische Aktivierung mit Hilfe von UVLicht (400 nm) die Sensitivität solcher Schichten steigern und in geringem Maße auch die Regenration beschleunigen kann [4]. Dieses empirische Ergebnis kann durch Vergleich mit bekannten Effekten aus der Photokatalyse besser verstanden und erklärt werden. Dadurch ist zudem eine weitere Optimierung möglich, wie im folgenden Artikel gezeigt wird. Das Schema in Abbildung 1 zeigt eine Auflistung grundlegender Effekte, die durch optische Aktivierung von halbleitenden Metalloxiden hervorgerufen werden können.

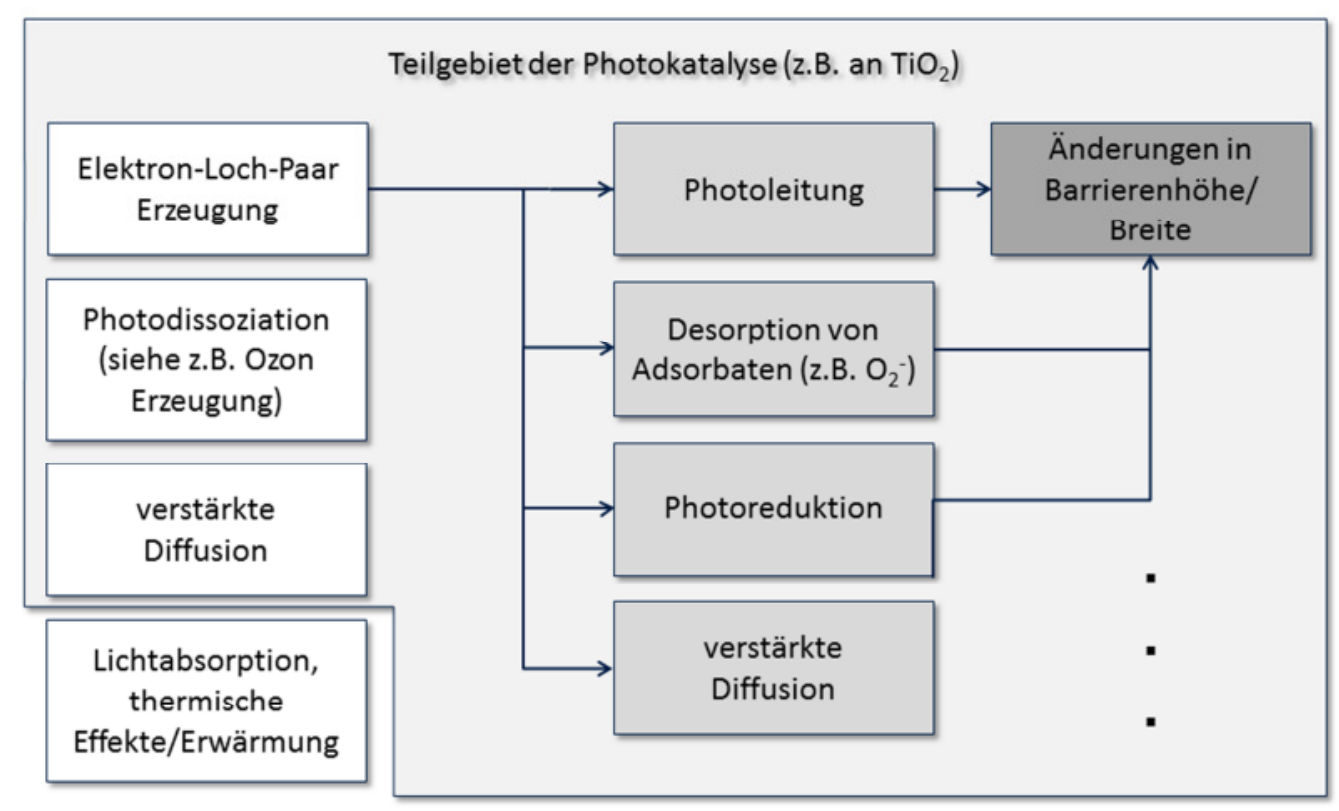

Abbildung 1: Auswahl grundlegender Effekte durch optische Aktivierung von halbleitenden Metalloxiden.

$\mathrm{Zu}$ Beginn fast aller für die Gassensorik relevanten Prozesse steht die Erzeugung von Elektronen-Loch-Paaren. Im Gegensatz zur sonst üblichen thermischen Aktivierung bei Temperaturen im typischen Bereich von $200-500{ }^{\circ} \mathrm{C}$ können Photonen selbst bei Weitband-Halbleitern Elektronen direkt vom Valenz- ins Leitungsband anregen. Für das weit verbreitete Zinnoxid $\left(\mathrm{SnO}_{2}\right)$ werden durch thermische Aktivierung typischerweise nur die beiden flachen Donatoren bei 30 
meV und $150 \mathrm{meV}$ [5] angeregt. Der Anteil an Elektronen direkt aus dem Valenzband (Bandlücke 3,6 eV) ist gering. Durch Bestrahlung mit UV-Licht (entspricht ca. $340 \mathrm{~nm}$ Wellenlänge) kann die dafür notwendige Energie jedoch leicht aufgebracht werden. Neben einer erhöhten Leitfähigkeit lösen die so erzeugten Elektronen-Loch-Paare wiederum weitere Effekte aus, von denen im Folgenden auf die Photo-Reduktion und die Aktivierung der Diffusion ionischer Spezies im Gitter näher eingegangen werden soll. Die Diffusion wird allerdings nicht nur durch Erzeugung von Elektronen-Loch-Paaren, sondern auch durch die direkte Energieübertragung auf die entsprechenden Ionen aktiviert. Im Folgenden werden diese beiden Prozesse nicht unterschieden, beide werden unter dem Begriff der optisch aktivierten Diffusion zusammengefasst.

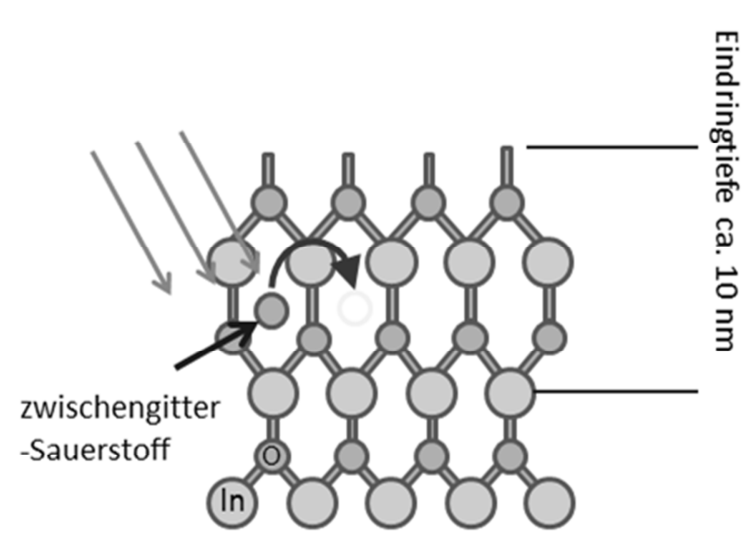

Abbildung 2: Schema zur optischen Aktivierung von Sauerstoffdiffusion auf Zwischengitterplätzen im $\operatorname{In}_{2} \mathrm{O}_{3}$-Gitter.

Bisherige Probleme, insbesondere bei der Signalstabilität, von $\operatorname{In}_{2} \mathrm{O}_{3}$-Sensoren bei Raumtemperatur, werden auf die langsame Sauerstoffdiffusion im Gitter zurückgeführt [6]. Sauerstofffehlstellen werden in dieser Arbeit als die Hauptursache für die relativ hohe Ladungsträgerkonzentration und damit den guten Leitwert von $\mathrm{In}_{2} \mathrm{O}_{3}$ trotz der großen optischen Bandlücke von ca. 3,6 eV angesehen. Die Aktivierungsenergie für die Diffusion im $\operatorname{In}_{2} \mathrm{O}_{3}$-Gitter beträgt ca. $1,72 \mathrm{eV}$ für Zwischengitterdiffusion und 1,92 eV für Diffusion über Sauerstoffdefekte [6]. Somit ist naheliegend, dass eine entsprechende Beleuchtung zu einer Beschleunigung der Diffusionseffekte führt. Es ist jedoch zu beachten, dass der Effekt durch die Eindringtiefe des verwendeten Lichtes begrenzt ist (siehe Abbildung 2). Für kompakte $\mathrm{In}_{2} \mathrm{O}_{3}$ Dünnschichten konnte eine Eindringtiefe von ca. 
$10 \mathrm{~nm}$ nachgewiesen werden [7]. Aus diesem Grund ist die Strukturierung der sensitiven Schichten auf den verschiedenen Größenskalen von besonderem Interesse [8]. Ähnliche Überlegungen gelten für die Photoreduktion. Durch Wechselwirkung mit einem Photon kann ein Sauerstoff 2p-Elektron in das Leitungsband gehoben werden, um anschließend in ein vakantes Orbital eines Metallatoms zu relaxieren. Durch diesen Prozess kann es zu einer Abspaltung von Sauerstoff und somit zu einer Reduktion des Metalloxids kommen. Als Daumenregel für die Photoreduzierbarkeit eines Oxids gilt: Ist die Abregungsenergie gröBer als die Bildungsenthalpie des neuen Oxids, dann ist der Prozess möglich [9]. Auch die Stärke des Photoreduktionseffektes wird durch die Struktur der Wirkschicht beeinflusst.

Im Folgenden werden Ergebnisse optisch aktivierter Sensormessungen mit geordnet mesoporösem $\operatorname{In}_{2} \mathrm{O}_{3}$ vorgestellt. Das Nanostrukturierte Material besitzt Strukturbreiten im Nanometerbereich und gewährt über das definierte Porennetzwerk guten Zugang für Gas und Licht.

\section{Experimentelles}

\subsection{Sensorherstellung}

Geordnet mesoporöses $\operatorname{In}_{2} \mathrm{O}_{3}$ wurde mit Hilfe des sogenannten nanocastingVerfahrens hergestellt. Mesoporöses KIT-6 Silika wird dabei durch Schmelzimprägnierung [10-12] mit Indiumnitrat imprägniert. Durch verschiedene Umsetzungsbedingungen (Variationen in der Gasatmosphäre) kann die Größe der strukturierten Partikel systematisch variiert werden.

Die Sensorschichten wurden durch Auftropfen wässriger Dispersionen (typ. $25 \mathrm{mg}$ Metalloxid in $1 \mathrm{~mL} \mathrm{H}_{2} \mathrm{O}$ dest.) auf kommerziell erhältliche Substrate der Firma Umweltsensortechnik GmbH hergestellt. Die Substrate haben eine Größe von $3 \times 3 \mathrm{~mm}^{2}$ und besitzen Interdigitalelektroden und einen integrierten Heizer (Pt10). Die Schichtdicken wurden mit Hilfe von REM Untersuchungen auf ca. $10 \mu \mathrm{m}$ geschätzt. Vor den ersten Messungen wurden die Sensoren für 24 Stun- 
den bei $175^{\circ} \mathrm{C}$ in Raumluft geheizt um Rückstände aus dem Herstellungsprozess zu entfernen.

\subsection{Strukturelle Charakterisierung}

Die Metalloxidproben wurden mit Hilfe von Pulver-Röntgendiffraktometrie (PXRD, Bruker AXS D8 Advance, $\mathrm{Cu}-\mathrm{K}_{\alpha}$ radiation), Stickstoff-Physisorption (Quantachrome NOVA 4000e and Autosorb 6 bei $77 \mathrm{~K}$; Proben wurden bei $120{ }^{\circ} \mathrm{C}$ für 24 Stunden entgast) und Rasterelektronenmikroskopie (Zeiss Neon 40) untersucht. Kristallitgrößen wurden mit Hilfe der Scherrer Methode und Porenradienverteilungen mit der BJH Methode berechnet.

\subsection{Gasmessungen}

Für die Gasmessungen wurden die Sensoren in einer speziell angefertigten Messzelle (PTFE) mit integrierter blauer LED (maximale Intensität bei $460 \mathrm{~nm}$ ) montiert. Die gewünschten Ozonkonzentrationen wurden mit Hilfe eines Computergesteuerten Gasmischaufbaus basierend auf Massendurchflussreglern in synthetischer Luft (10\% rel. Feuchte) eingestellt. Das Ozon wurde mit einem kommerziell erhältlichen Generator (UVP Modell SOG-1) erzeugt und mit einem Ozon-Monitor (2B Technologies Modell 202) überwacht. Die Variation der Ozonkonzentration erfolgte durch Variation der Lichtintensität am Ozongenerator.

Die Widerstandsänderungen der sensitiven Schichten wurden mit einem Digitalmultimeter (Agilent 34972A) mit 16-Kanal Multiplexer (Agilent 34902A) aufgenommen. Die automatische Messbereichsumschaltung wurde für die Raumtemperaturmessungen deaktiviert, um den Einfluss verschiedener Messströme auf das Sensorsignal auszuschließen.

Die Messungen wurden bei Raumtemperatur durchgeführt. Jeder Sensor durchlief vor einer erneuten Messung eine reset-Prozedur. Durch eine Wärmebehandlung für 10 Minuten bei $100{ }^{\circ} \mathrm{C}$ mit Hilfe des eingebauten Heizers in synthetischer Luft und gleichzeitiger Beleuchtung durch die LED wurden die Sensoren in einen definierten Startzustand überführt. 


\section{Ergebnisse und Diskussion}

\subsection{Materialcharakterisierung}
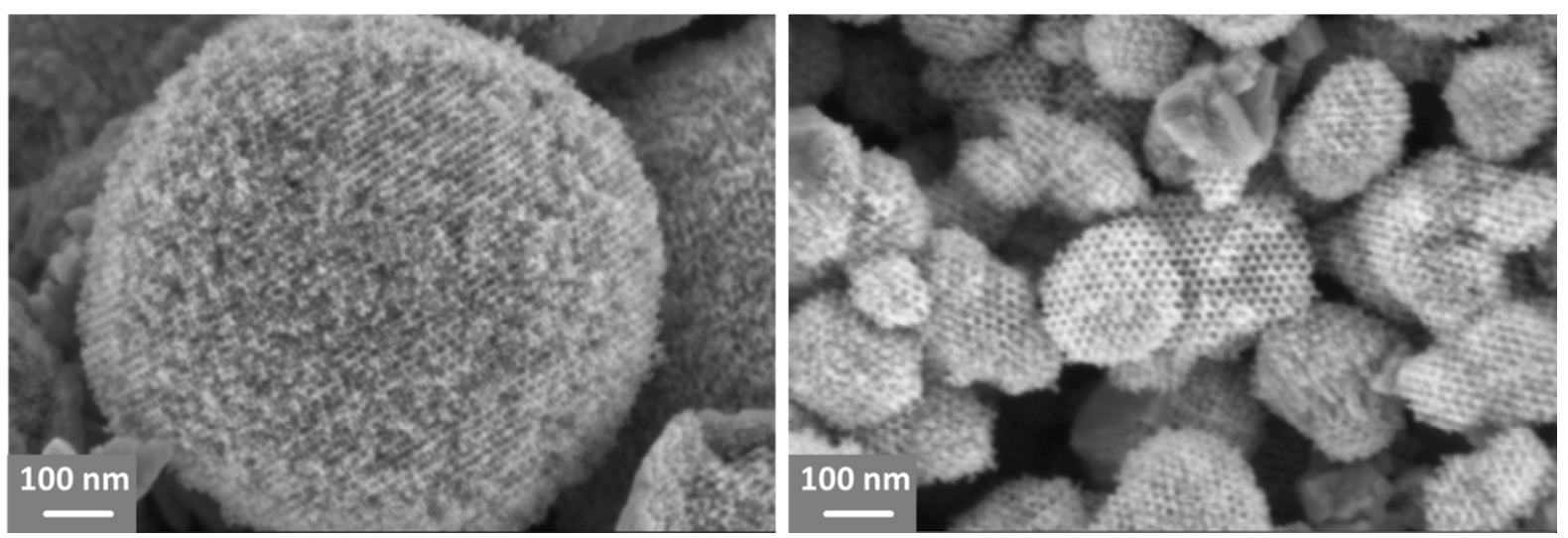

Abbildung 3: REM Aufnahmen der geordnet mesoporösen $\operatorname{In}_{2} \mathrm{O}_{3}$-Partikel verschiedener Größe.

Abbildung 3 zeigt elektronenmikroskopische Aufnahmen (REM) der geordnet mesoporösen $\mathrm{In}_{2} \mathrm{O}_{3}$-Proben mit verschiedenen Partikelgrößen. Die poröse Struktur der Partikel ist deutlich zu erkennen. Eine detaillierte Beschreibung des Herstellungsprozesses und der Probenparameter findet sich an anderer Stelle [10]. Tabelle 1 fasst die Ergebnisse der Charakterisierung zusammen.

\begin{tabular}{ccc} 
& große $\mathrm{In}_{2} \mathrm{O}_{3}$ Partikel & kleine $\mathrm{In}_{2} \mathrm{O}_{3}$ Partikel \\
\hline $\begin{array}{c}\text { durchschnittlicher } \\
\text { Partikeldurchmesser } \\
\text { Porenradius }\end{array}$ & $870 \mathrm{~nm}$ & $170 \mathrm{~nm}$ \\
$\begin{array}{c}\text { spez. Oberfläche } \\
\text { (BET) }\end{array}$ & $4 \mathrm{~nm},(5 \mathrm{~nm})$ & $4 \mathrm{~nm}, 5 \mathrm{~nm}, 12 \mathrm{~nm}$ \\
& $46 \mathrm{~m}^{2} / \mathrm{g}$ & $101 \mathrm{~m}^{2} / \mathrm{g}$
\end{tabular}

Tabelle 1: Zusammenfassung der Strukturparameter der kleinen und großen $\mathrm{In}_{2} \mathrm{O}_{3}$ Partikel. [10]

Es ist zu bemerken, dass die verschiedenen Umwandlungsmethoden neben der Partikelgröße auch zu verschiedenen Porenradienverteilungen führen. Die kleinen Partikel besitzen zusätzlich 12 nm Poren, die auf Grund unvollständiger Füllung der Poren während des nanocasting-Verfahrens zurückzuführen sind. Für 
die folgenden Überlegungen wird dieser Effekt im Vergleich zur Änderung der Partikelgröße als untergeordnet angenommen.

\subsection{Ozonmessungen}

Messungen mit verschiedenen Ozon-Konzentrationen von ca. $50 \mathrm{ppb}$ bis $\mathrm{zu}$ $220 \mathrm{ppb}$ zeigen eine starke und schnelle Reaktion beider Materialien (siehe Abbildung 4 , links). Die $t_{90}$ Zeiten (Zeit bis zum Erreichen von $90 \%$ des maximal erreichten Signals) für die sensitiven Schichten mit den großen Partikeln können nicht ermittelt werden, da in der Messzeit kein stabiler Wert erreicht wurde. Für die kleinen Partikel jedoch kann ein Wert von ca. 4 Min. angegeben werden. Diese Zeiten liegen deutlich unter den Zeiten, die für unbeleuchtete $\operatorname{In}_{2} \mathrm{O}_{3^{-}}$ Proben gefunden wurden (34 Min. bei 1,2 ppm Ozon [13]). Dieser Unterschied zwischen den Proben war zu erwarten, da die Eindringtiefe des zur Aktivierung genutzten Lichtes begrenzt ist.
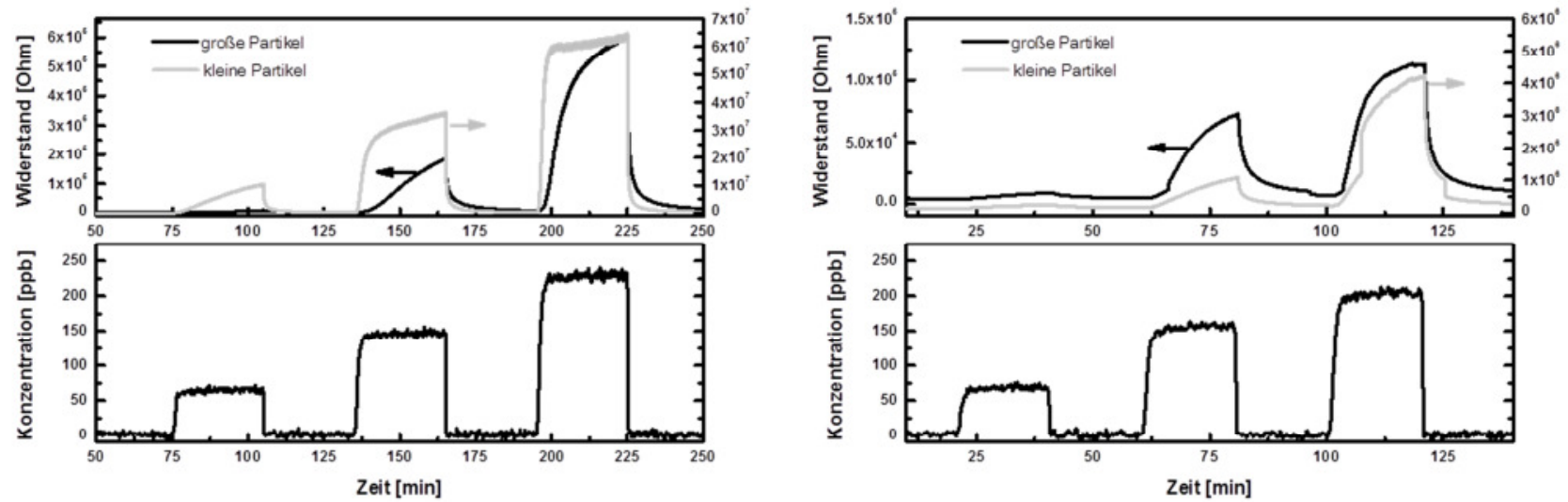

Abbildung 4: Links: Vergleich einer Raumtemperaturmessung unter Beleuchtung mit großen und kleinen $\operatorname{In}_{2} \mathrm{O}_{3}$-Partikeln in der Wirkschicht [14]; Rechts: Messung mit Beleuchtung und $100^{\circ} \mathrm{C}$ Betriebstemperatur.

Die großen Partikel werden nicht vollständig aktiviert und es kommt zu einer Mischung aus aktivierter und nicht aktivierter Sauerstoffdiffusion im Gitter, die zu einer langsameren Stabilisierung des Signals führt. Werden die Proben während der Messung zusätzlich beheizt, verschwindet dieser Unterschied im Zeitverhalten (siehe Abbildung 4, rechts). Dies ist zu erwarten, da die thermische Aktivierung auf die gesamte Probe wirkt. 
Die Regeneration der Sensoren mit kleinen Partikeln nach Abschalten des Ozonangebots erfolgt ebenfalls schneller verglichen mit den Wirkschichten aus großen Partikeln, jedoch ist hier der Unterschied nicht so ausgeprägt. Nach ca. 1 Min. erreichen die kleinen Partikel $10 \%$ des Maximalsignals; die großen Partikel benötigen ca. 3,5 Min.. Dies hängt unter anderem mit der Eindringtiefe des Ozons in die porösen Partikel zusammen. Eine detailliertere Beschreibung findet sich an anderer Stelle [14]. Die Regenration wird von zwei Effekten dominiert. Zum einen kommt es wieder zu einer beschleunigten Diffusion des Sauerstoffs im Kristallgitter. Zum anderen führt die Beleuchtung zu einer Photoreduktion des $\mathrm{In}_{2} \mathrm{O}_{3}$ und damit zur Widerherstellung des Gleichgewichts zwischen Sauerstoffkonzentration im Gitter und der Umgebungsluft. Beide Effekte sind durch die Eindringtiefe des Lichts begrenzt.

In vorherigen Arbeiten konnte zudem gezeigt werden, dass der Dynamikbereich der Sensoren durch optische Aktivierung vergrößert werden kann. Durch Photoreduktion verschiebt sich das Reaktionsgleichgewicht zwischen Sauerstoffaufnahme in das $\operatorname{In}_{2} \mathrm{O}_{3}$-Gitter bei Ozonangebot und der Desorption in Richtung Desorption. Dadurch kommt es erst wesentlich später zu einer Sättigung des Gitters bzw. einer Erschöpfung der Sauerstofffehlstellen im Gitter. Für vergleichbare Proben wie die hier gezeigten, konnte ohne Beleuchtung für 1,2 ppm Ozonangebot eine Sensitivität (Schichtwiderstand bei Gasangebot / Widerstand in synth. Luft) von 72,2 und bei bei 2,4 ppm Ozon von 62,3 ermittelt werden [13]. Der Film war also bereits gesättigt. Mit Beleuchtung betrugen die Werte 353,26 bzw. 1764,6.

Da die zuvor beschriebenen Effekte den Ein- und Ausbau von Sauerstoff in das Metalloxidgitter beinhalten, wurde außerdem die Zyklenstabilität getestet (siehe Abbildung 5). Wie sich zeigt, kommt es bei den Wirkschichten mit kleinen Partikeln zu einer erheblichen Drift bei mehreren kurz aufeinander folgenden Gasangeboten. Dies wird darauf zurückgeführt, dass sich die Fehlstellenkonzentration im Metalloxidgitter der kleinen Partikel schneller an die mittlere Sauerstoffkonzentration (Ozon) in der Umgebung anpasst. Erst eine Regeneration durch zusätzliches Ausheizen bzw. eine längere Wartezeit als die im Zyklentest verwendeten 20 Minuten führt zu einer Regeneration. Die großen Partikel hingegen werden durch die zyklische Beaufschlagung weniger stark beeinflusst, der nicht 
von den Beleuchtungseffekten betroffene Kern der Partikel stellt somit eine Art Puffer für die Fehlstellen dar [15].

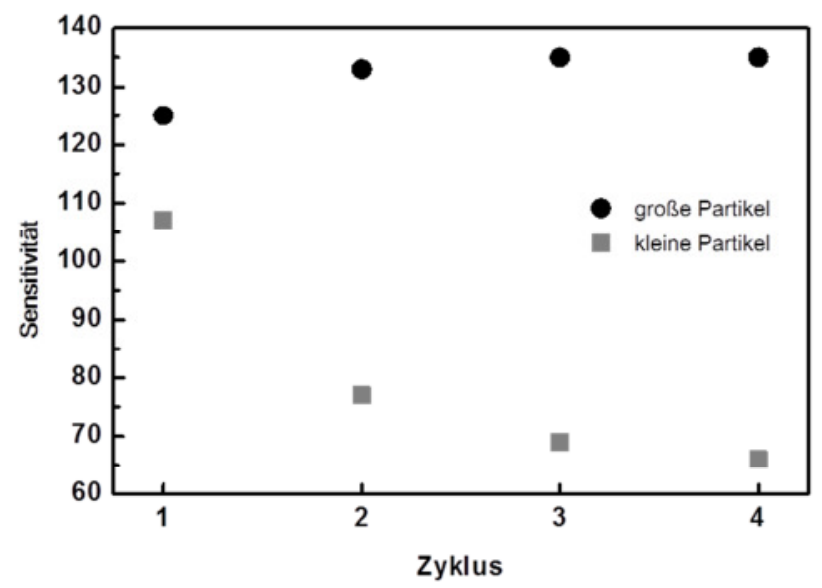

Abbildung 5: Test zur Zyklenstabilität: die Sensoren wurden zyklisch für 20 min. Ozon-Konzentrationen von 220 ppb ausgesetzt; zwischen den Gasangeboten wurden die Sensoren 20 min. in synthetischer Luft regeneriert.

\section{Fazit}

Durch optische Aktivierung ist es möglich, halbleiterbasierte Gassensoren für den Betrieb bei Raumtemperatur (für reaktive Gase) herzustellen. Um die durch die optische Aktivierung generierten Effekte nutzen zu können, muss die Nanound Mikrostruktur der Wirkschicht den Anforderungen an die jeweilige Messaufgabe angepasst werden. Im vorliegenden Fall konnte gezeigt werden, dass z.B. geordnet mesoporöse Materialien gut für die optische Aktivierung geeignet sind. Auf Grund der porösen Struktur (Nanostruktur) und den Wandstärken im Nanometerbereich erreicht das Licht eine gute Durchdringung der Strukturen und ermöglicht somit eine schnelle Stabilisierung der Signale. Durch Variation der Partikelgröße (Mikrostruktur) kann weiterhin Einfluss auf die Reaktionsund Regenerationszeiten genommen werden. Die kleineren Partikel reagieren schneller, zeigen jedoch bei zyklischer Gasaufgabe Drifteffekte. Somit muss die Mikrostruktur ebenfalls für den jeweiligen Messbetrieb angepasst werden. 


\section{Danksagung}

Wir bedanken uns bei der Deutschen Forschungsgemeinschaft für die finanzielle Unterstützung.

\section{Literatur}

[1] Takada, T., Suzuki, K., Nakane, M., Sensors and Actuators B: Chemical 13, 404-407 (1993).

[2] Gurlo, A., Barsan, N., Ivanovskaya, M., Weimar, U., Göpel, W., Sensors and Actuators B: Chemical 47, 92-99 (1998).

[3] Ivanovskaya, M., Gurlo, A., Bogdanov, P., Sensors and Actuators B: Chemical 77, 264-267 (2001).

[4] Wang, C.Y., Cimalla, V., Kups, T., Rohlig, C., Stauden, T., Ambacher, O., Kunzer, M., Passow, T., Schirmacher, W., Pletschen, W.; et al., Applied Physics Letters 91, 103509-3 (2007).

[5] Samson, S., Fonstad, C.G., J. Appl. Phys. 44, 4618-4621 (1973).

[6] Wirtz, G.P., Takiar, H.P., Journal of the American Ceramic Society 64, 748-752 (1981).

[7] Bender, M., Katsarakis, N., Gagaoudakis, E., Hourdakis, E., Douloufakis, E., Cimalla, V., Kiriakidis, G., J. Appl. Phys. 90, 5382-5387 (2001).

[8] Wagner, T., Donato, N., Tiemann, M., New Sensing Model of (Mesoporous) $\mathrm{In}_{2} \mathrm{O}_{3}$ in: C.-D. Kohl, T. Wagner (Hrsg.), Springer Series on Chemical Sensors and Biosensors (ISSN: 1612-7617), Springer, Berlin/Heidelberg (2014)

[9] Fleisch, T.H., Zajac, G.W., Schreiner, J.O., Mains, G.J., Applied Surface Science 26, 488-497 (1986).

[10] Klaus, D., Amrehn, S., Tiemann, M., Wagner, T., Microporous and Mesoporous Materials 188, 133-139 (2014).

[11] Haffer, S., Weinberger, C., Tiemann, M., Eur. J. Inorg. Chem. 2012, 3283-3288 (2012).

[12] Weinberger, C., Haffer, S., Wagner, T., Tiemann, M., Eur. J. Inorg. Chem. 2014, 2787-2792 (2014).

[13] Wagner, T., Hennemann, J., Kohl, C.-D., Tiemann, M., Thin Solid Films 520, 918-921 (2011).

[14] Klaus, D., Klawinski, D., Amrehn, S., Tiemann, M., Wagner, T., Sensors and Actuators B: Chemical; eingereicht.

[15] Wagner, T., Kohl, C.-D., Morandi, S., Malagù, C., Donato, N., Latino, M., Neri, G., Tiemann, M., Chemistry - A European Journal 18, 82168223 (2012). 